\title{
Phenotypes of hypertrophic cardiomyopathy. An illustrative review of MRI findings
}

\author{
Rafaela Soler ${ }^{1} \cdot$ Cristina Méndez $^{1} \cdot$ Esther Rodríguez $^{1} \cdot$ Roberto Barriales $^{2} \cdot$ Juan Pablo Ochoa $^{2} \cdot$ Lorenzo Monserrat $^{2}$
}

Received: 16 April 2018 /Revised: 6 July 2018 / Accepted: 28 August 2018 / Published online: 22 October 2018

(C) The Author(s) 2018

\begin{abstract}
Objective The purpose of this article is to review how cardiac MRI provides the clinician with detailed information about the hypertrophic cardiomyopathy (HCM) phenotypes, assessing its morphological and functional consequences.

Conclusion An understanding of cardiac MRI manifestations of HCM phenotypes will aid early diagnosis recognition and its functional consequences.

Teaching Points

- The phenotypic variability of HCM expands beyond myocardial hypertrophy, to include morphological and functional manifestations, ranging from subtle anomalies to remodelling of the $L V$ with progressive dilatation and thinning of its wall.

- The stages of HCM, which are based on the clinical evidence of disease progression, include subclinical HCM, the classic HCM phenothype, adverse remodelling and overt dysfunction, or end-stage HCM.

- Cardiac MRI provides the clinician with detailed information regarding the HCM phenotypes and enables the assessment of its functional consequences.
\end{abstract}

Keywords Hypertrophic cardiomyopathy $\cdot$ Phenotypes $\cdot$ Magnetic resonance $\cdot$ Heart

$\begin{array}{ll}\text { Abbreviations } \\ \text { ECV } & \text { Extracellular volume } \\ \text { EF } & \text { Ejection fraction } \\ \text { HCM } & \text { Hypertrophic cardiomyopathy } \\ \text { ICD } & \text { Implantable cardioverter defibrillator } \\ \text { LA } & \text { Left atrium } \\ \text { LGE } & \text { Late-gadolinium enhancement } \\ \text { LV } & \text { Left ventricle } \\ \text { LVOT } & \text { LV outflow tract } \\ \text { MRI } & \text { Magnetic resonance imaging } \\ \text { PET } & \text { Positron emission tomography } \\ \text { RV } & \text { Right ventricle }\end{array}$

Esther Rodríguez

estrodrigarci@gmail.com

1 Radiology Department, Complexo Hospitalario Universitario A Coruña, Instituto de Investigación Biomédica de A Coruña (INIBIC), Servizo Galego de Saúde (SERGAS), Universidade da Coruña, Xubias de Arriba 86, 15006 A Coruña, Spain

2 Cardiology Department, Complexo Hospitalario Universitario A Coruña, Instituto de Investigación Biomédica de A Coruña (INIBIC), Servizo Galego de Saúde (SERGAS), Universidade da Coruña, Xubias de Arriba, 84, 15006 A Coruña, Spain
SAM Systolic anterior motion

SCD Sudden cardiac death

SPECT Single photon emission computed tomography

\section{Introduction}

Hypertrophic cardiomyopathy (HCM) is a genetic cardiovascular disease, defined by an increase in the left ventricular wall thickness (end-diastolic left ventricular wall thickness $\geq 15 \mathrm{~mm}$ or the equivalent relative to the body surface area in children) that is not solely explained by abnormal loading conditions. Lesser degrees of wall thickness (13-14 $\mathrm{mm})$ can also be diagnostic of HCM, particularly when it is identified in family members [1-3]. In up to $60 \%$ of adolescents and adults, this disorder is an autosomal dominant trait caused by mutations in cardiac sarcomere protein genes [2, 4, 5].

The microscopic findings of HCM are characteristic with hypertrophy of the myocardial fibres, disorganisation of muscular bundles, interstitial fibrosis and dysfunction of the coronary microvasculature with increased wall thickening that leads to luminal narrowing, silent myocardial ischaemia, and myocardial injury and fibrosis [6]. 
HCM is characterised by diverse phenotypic expressions and a variable natural progression, which may range from dyspnoea and/or syncope to sudden cardiac death (SCD). HCM is the most common cause of SCD in young athletes $[2,3,7,8]$.

The phenotypic variability of HCM is not limited to only myocardial hypertrophy, but rather includes a set of morphological and functional manifestations, ranging from subtle anomalies to remodelling of the left ventricle (LV) with progressive dilatation and thinning of its wall that evolves to heart failure simulating a restrictive or dilated cardiomyopathy [8-12]. It is important to look for each of these phenotypic expressions to establish the diagnosis and define the importance of the disease [2,8].

Cardiac MRI has emerged as an imaging modality particularly well-suited to characterise the phenotypic expression of $\mathrm{HCM}$, providing more accurate wall thickness measurements and identifying patients with an increased risk for ventricular arrhythmias and thromboembolic stroke, as well as abnormalities that contribute to LV outflow tract (LVOT) obstruction and differentiating HCM from other causes of LV hypertrophy $[3,8,13]$.

Additionally, contrast-enhanced cardiac MR with lategadolinium enhancement (LGE) has the ability to identify areas of myocardial fibrosis/scarring that may be at increased risk for $\mathrm{SCD}$, and it has implications for management strategies, such as primary prevention implantable cardioverter defibrillator (ICD) therapy $[3,8,13-15]$.

The stages of HCM, which are based on the clinical evidence of disease progression, were previously defined by Olivotto et al. [16]. These clinical stages include subclinical HCM, the classic HCM phenothype, adverse remodelling and overt dysfunction, or end-stage HCM.

The aim of this article is to illustrate and review the contributions of cardiac MRI for the assessment of morphological and functional consequences of different stages of $\mathrm{HCM}$ (Table 1).

\section{Subclinical HCM}

Subclinical HCM refers to the detection of subjects who carry any stigmata of HCM causing gene mutation but who are without LV hypertrophy (genotype-positive, phenotypenegative subjects). In these patients, the risk for SCD is exceptional [9].

When genetic testing is negative or ambiguous (30 to $40 \%$ of patients), cardiac MR can demonstrate a degree of LV hypertrophy that is not fully appreciated by echocardiogram [8] and other abnormalities, such as myocardial crypts, elongated mitral valve leaflets and apical myocardial trabeculations [8-13], expanded extracellular space (with T1 mapping) and LGE $[17,18]$. These individuals should be periodically followed for the development of an increased LV wall thickness $[4,5,19]$.

\section{Myocardial crypts}

Myocardial clefts or crypts are congenital abnormalities related to myocardial fibre or fascicle disarray and have been described in healthy volunteers, as well as those with classic $\operatorname{HCM}(<5 \%)[8,20,21]$.

These crypts are V- or U-shaped blood-filled myocardial invaginations perpendicular to the endocardial LV edge that penetrate more than $50 \%$ of the compact myocardium at enddiastole and collapse at end-systole (Fig. 1) [10, 21, 22]. Usually, myocardial crypts are multiple and located in the basal inferoseptal and inferior LV wall at the sites of right ventricle (RV) insertion $[9,22]$.

These myocardial crypts should be differentiated from trabeculations characteristic of LV noncompaction, congenital LV diverticula and aneurysms.

Unlike crypts, trabeculations of LV noncompaction are parallel to the endocardial border and do not penetrate the normal compacted myocardium [22].

Congenital LV diverticula are characterised by an outpouching that contains endocardium, myocardium, and pericardium, and they are a narrow connection to the cavity and collapse at end-systole. The primary diagnostic feature to differentiate diverticula from crypts is that the congenital diverticula extend outside the epicardial border while crypts remain confined inside the myocardial margin [23].

Congenital LV aneurysm represents an outpouching containing endocardium, epicardium and thinned myocardium that shows akinetic or dyskinetic motion. A thinned myocardium shows LGE without pericardial enhancement [23, 24].

\section{Mitral valve leaflets elongation}

Several echocardiographic studies have reported that the mitral valve is elongated in HCM, particularly in the obstructive form of the disease [25, 26].

Cardiac MR studies have shown elongated anterior and posterior mitral valve leaflets in patients with non-genotyped HCM compared to control subjects [11] and elongated anterior mitral valve leaflets in subjects with HCM-causing mutations without LV hypertrophy (Fig. 2) [11, 12, 27]. These studies suggest that anterior mitral valve leaflet elongation in young preclinical HCM patients without other phenotypic expressions of HCM represents an independent and primary component of HCM disease expression independent of age, LV thickness or the presence of LV outflow tract obstruction.

Other recent studies suggest that mitral valve elongation does not constitute the primary phenotypic expression of $\mathrm{HCM}$ and that the elongation of mitral valve leaflets in 
Table 1 Cardiac MR characteristics of different stages of HCM

Cardiac MR imaging

\begin{tabular}{|c|c|c|c|c|c|}
\hline \multirow[b]{2}{*}{ HCM stage } & \multirow[b]{2}{*}{ Morphological and functional features } & \multicolumn{4}{|c|}{ Late-gadolinium enhancement (LGE) } \\
\hline & & Prevalence & $\% / \mathrm{LV}$ mass & Location & $\mathrm{SCD}$ risk/year \\
\hline Subclinical & $\begin{array}{l}\text { Normal/borderline myocardial hypertrophy } \\
\text { Myocardial crypts } \\
\text { Mitral valve leaflets elongation } \\
\text { Normal LA diameter } \\
\text { Myocardial trabeculations } \\
\text { Myocardial fibrosis } \\
\text { Normal LV EF }\end{array}$ & Rare & Limited & Mid-wall & Exceptional \\
\hline Classic $(75 \%)$ & $\begin{array}{l}\text { Myocardial hypertrophy } \\
\text { LVOT obstruction ( } 70 \% \text { ) } \\
\text { Mid or moderate LA dilatation } \\
\text { Diastolic dysfunction } \\
\text { LV EF }>65 \%\end{array}$ & $<50 \%$ & $2 \%$ & Mid-wall & $0,5-1 \%$ \\
\hline Adverse remodelling (15-20\%) & $\begin{array}{l}\text { Progressive LV wall thinning } \\
\text { Reduction/loss of LVOT } \\
\text { Moderate/severe LA dilatation } \\
\text { Diastolic dysfunction } \\
\text { LV EF: } 50-65 \%\end{array}$ & $>50 \%$ & $10-15 \%$ & Mid-wall/transmural & $3-5 \%$ \\
\hline End-stage $(5-10 \%)$ & $\begin{array}{l}\text { Dilated form } \\
\text { Restrictive pattern } \\
\text { LV EF }<50 \%\end{array}$ & $75-100 \%$ & $>25 \%$ & Mid-wall/transmural & $10 \%$ \\
\hline
\end{tabular}

$L V$ left ventricle, $E F$ ejection fraction, $S C D$ sudden cardiac death, $L A$ left atrium, $L V O T$ left ventricular outflow tract

HCM seems to be associated with body size and left ventricular remodelling [28].

\section{Myocardial trabeculations}

Left ventricular non-compaction is a genetically heterogeneous disorder and shares genetic mutations with HCM. Both entities can coexist and have been described as being associated with mutations in different sarcomeric genes [29].

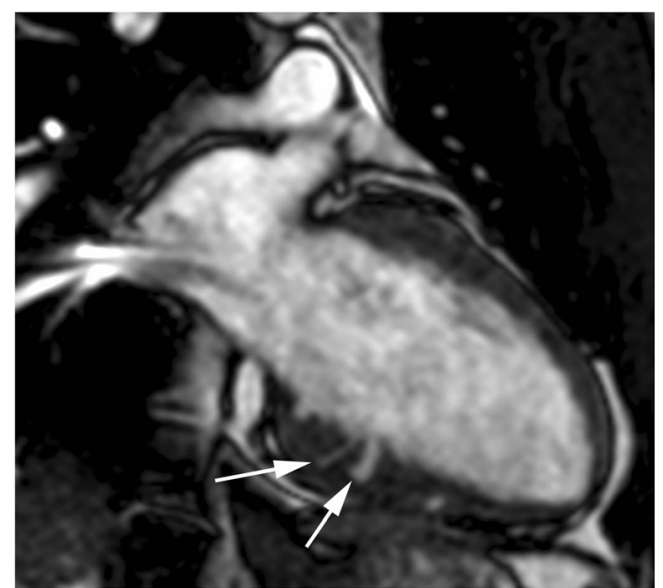

Fig. 1 22-year-old man with myocardial crypts and a family history of HCM without myocardial hypertrophy. Two-chamber steady-state free precession (SSFP) cine MR image at end-diastole shows V-shaped myocardial crypts perpendicular to the endocardial border at inferior LV wall (arrows). The crypts close completely during systole (not shown)
Abnormal LV apical myocardial trabeculations also can be detected in HCM sarcomere gene mutation carriers without evident hypertrophy (Fig. 3). Both diseases can have the same mutation of myocardial sarcomere, and they are indistinguishable [12]. The mechanism for these abnormal trabeculations remains unclear, similar to myocardial crypts, they could represent the persistence of the embryological form into adulthood $[10,12]$.

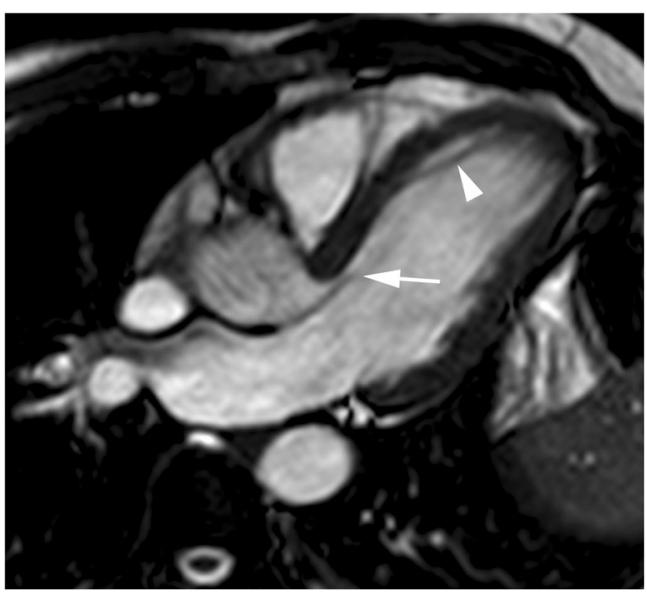

Fig. 2 20-year-old man, genotype positive, phenotype negative. Enddiastolic horizontal long-axis SSFP cine MR image demonstrates an elongated anterior mitral valve leaflet (arrow) and normal length of posterior valve leaflet. A linear structure (arrowhead) that connects ventricular septum and left ventricular apex, corresponding to aberrant muscular band also can be seen 


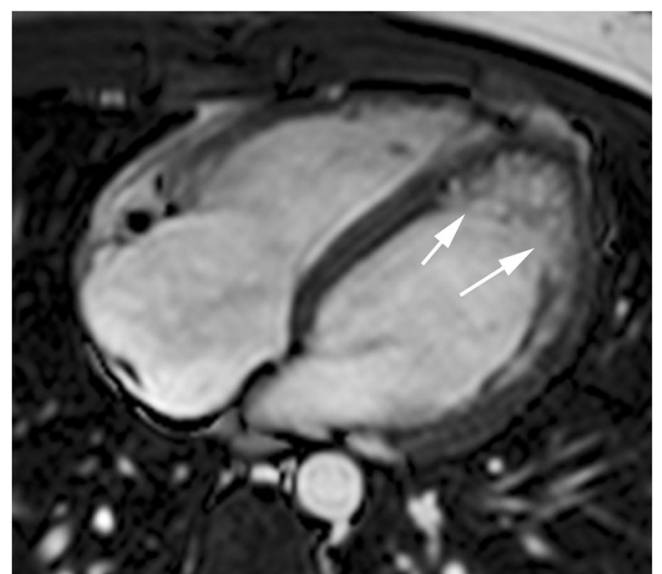

Fig. 3 22-year-old man with a family history of HCM, genotype positive, phenotype negative. End-diastolic SSFP cine MR images showing prominent trabeculations in the LV apex (arrows) and normal miocardial thickness. These MRI features are indistinguishable from LV non-compaction

\section{Myocardial fibrosis}

The development of fibrosis is an important step in the LV remodelling process of HCM. Recent studies have demonstrated that HCM genotype-positive and phenotype-negative patients have increased levels of biomarkers of collagen deposition. This finding indicates that increased levels of profibrotic markers are also found in patients without ventricular hypertrophy, suggesting that hypertrophy is preceded by the fibrosis process. In genotype-positive, phenotype-negative HCM subjects, several cardiac MR studies found that the myocardial mid-wall LGE consistent with fibrosis was attributed to episodes of myocardial ischaemia due to coronary microcirculation remodelling [18]. Other studies have shown that both native T1 values and extracellular volume (ECV) fraction obtained after gadolinium administration can detect diffuse interstitial fibrosis and are elevated in regions without LGE in subjects with a mutation known to cause HCM without sufficient phenotypic expression to diagnose the disease based on current imaging criteria [17].

Native T1 mapping and the ECV fraction could be used at the initial phase of HCM for the early detection of interstitial diffuse myocardial fibrosis in these patients and to monitor disease progression, as well as to evaluate novel diseasemodifying therapy, targeting interstitial fibrosis $[8,17]$.

\section{Differential diagnosis}

The diagnosis of mild HCM in young competitive athletes may be challenging when the LV wall thickness is between 13 and $15 \mathrm{~mm}$ (or 12 and $13 \mathrm{~mm}$ in women), which identifies the "grey-zone" of overlap between the physiological adaptations to training and mild phenotypic expression of the disease. In such instances, the diagnosis can often be resolved by applying noninvasive markers. $\mathrm{HCM}$ is favoured with the identification of a pathogenic sarcomere mutation or a family history of HCM, an abnormal LV filling/relaxation, small/normal size LV, myocardial crypts or LGE on contrast cardiac MR and response to detraining $[30,31]$.

Recent studies have shown that native $\mathrm{T} 1$ values and myocardial ECV by T1 mapping can be used in the differential diagnosis between HCM and an athlete's heart. While the ECV fraction increases with increasing LV hypertrophy in HCM (due to extracellular matrix expansion and myocardial disarray), the ECV fraction reduces in athletes with an increasing wall thickness (due to an increase in the healthy myocardium by cellular hypertrophy) [32].

\section{Classic HCM}

The classic and most common HCM phenotype (75\%) consists of a hypertrophied, nondilated and hyperdynamic LV (ejection fraction $>65 \%$ ), which arises during adolescence and is usually complete by young adulthood, though the onset of its phenotype may occur at virtually any age, including in utero and in those older than 60 years [33].

\section{Distribution of hypertrophy}

The distribution of myocardial hypertrophy is extremely variable. Cardiac MRI is particularly useful for characterising the location and extent of LV hypertrophy (Fig. 4), offering a superior visualisation and a higher diagnostic accuracy compared with 2D echocardiography, particularly if the involved segments are the basal anterolateral free wall or the apex [34, 35].

\section{Left ventricular hypertrophy}

The most common location of LV hypertrophy is the confluence of the basal anterior septum with the contiguous anterior free wall $(70 \%)$ (Fig. 4b) [3, 8, 35]. The next most common segment with increased wall thickening is the posterior septum at the mid-LV level $[27,35]$.

The extent of hypertrophy is focal (involving fewer than three segments), intermediate (3-7 segments) or most common diffuse (more than seven segments) (Fig. 4a). Patients with LVOT obstruction shows hypertrophy of more segments [35]. Massive hypertrophy is considered when the LV wall thickness is $>3 \mathrm{~cm}$ and it has important prognostic considerations. However, in approximately $20 \%$ of HCM patients, the LV mass index values are normal on cardiac MRI $[8,19]$.

Mid-ventricular HCM shows predominant hypertrophy at the mid-ventricular level. Patients with this type of HCM constitute an unusual and important group of 

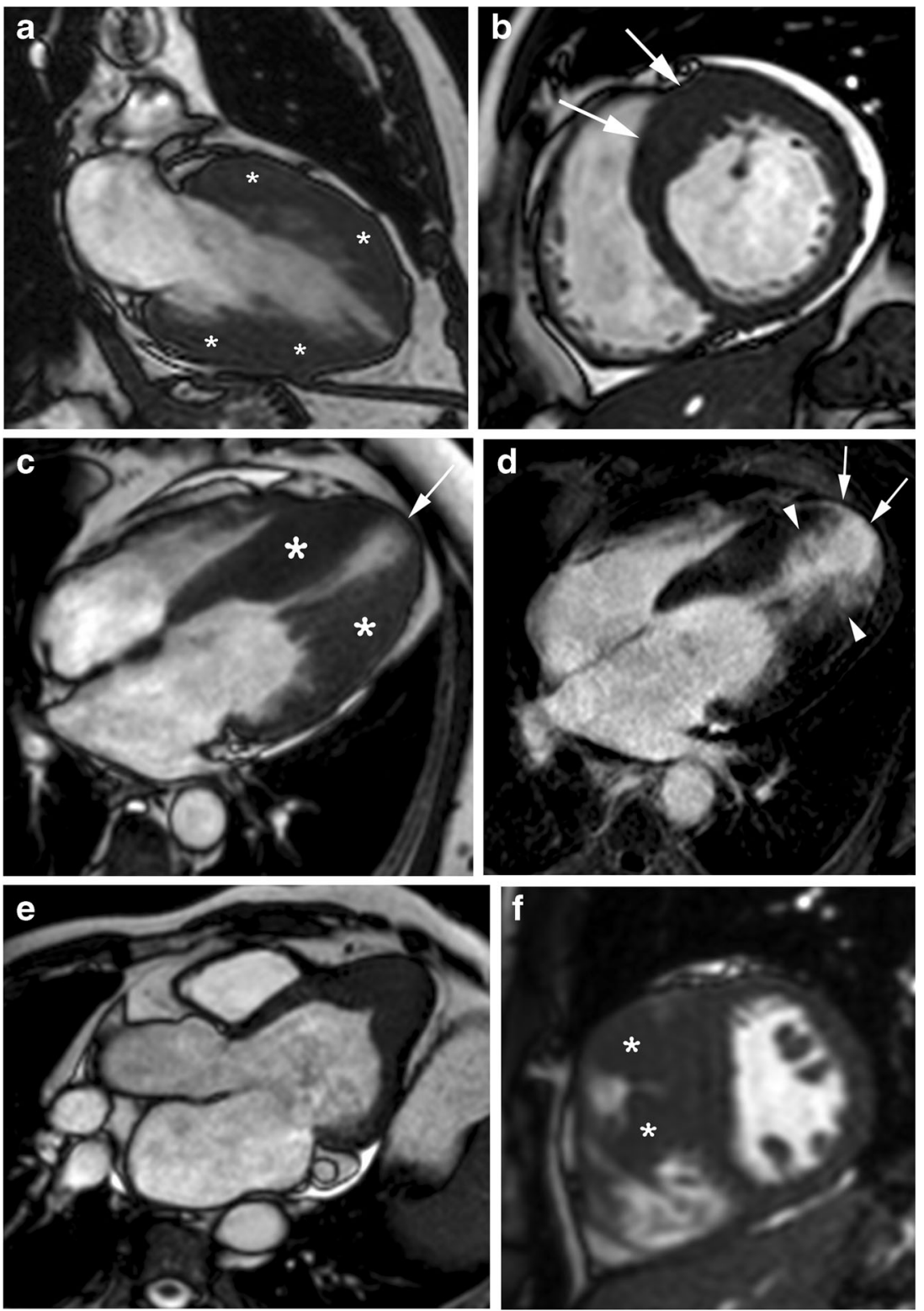

Fig. 4 Distribution of myocardial hypertrophy in classic HCM phenotype. a, 56-year-old man with symmetric myocardial hypertrophy. End-diastolic two-chamber SSFP cine MR image shows diffuse and symmetric LV wall hypertrophy (asterisks); b, 43year-old man who presented with palpitations. Short-axis SSFP cine MR image at end-diastole shows asymmetric septal wall hypertrophy (arrows); c, 57-year-old man with severe dyspnea. Enddiastolic four-chamber SSFP cine MR image demonstrates a "dumbbell" configuration of the LV cavity with midventricular myocardial thickening (asterisks), marked muscular midcavity systolic constriction and a thin-walled apical aneurysm (arrow). d,

patients because severe mid-ventricular HCM can cause ventricular arrhythmia, myocardial necrosis, and systemic embolism secondary to dynamic obstruction associated with apical aneurysm (Fig. 4c) [3, 36]. Contrastenhanced cardiac MR has demonstrated that the aneurysm rim in these patients is composed predominantly of fibrosis that extends from the aneurysm rim into the septum
Four-chamber LGE MR image clearly depicts the enhanced thinned apical LV aneurysm (arrows) extending from the aneurysm rim into the septum and free wall (arrowheads). Global systolic function also decreased because the ejection fraction was 35\%; e, 53-yearold woman with apical HCM. End-diastolic horizontal long-axis SSFP MR image demosntrates the spade-like appearance of LV chamber secondary to apical LV hypertrophy; f, 22-year-old man with right ventricular hypertrophy. Short-axis SSFP cine MR image at end-diastole shows a prominent right ventricular structure that inserts directly on the anterior ventricular septum corresponding to large hypertophied crista supraventicularis muscle (arrowheads)

and free wall (Fig. 4d) and serves as nidus for ventricular tachycardia [36].

Apical HCM (5-25\%) shows a predominant apical distribution of hypertrophy with a characteristic spade-like configuration of the LV cavity and is usually associated with giant inverted anterolateral T-waves on the electrocardiogram (Fig. 4e) [34]. The apical extension of LV hypertrophy 
is found in $10 \%$ of classic septal HCM patients, suggesting that both phenotypic expressions may coexist [8].

\section{Right ventricular hypertrophy}

Hypertrophy of the RV wall and RV muscle structures, such as that of the crista supraventricularis are common (Fig. 4f). Most HCM patients (53\%) have diffuse RV hypertrophy involving all three segments of the RV, but a conspicuous proportion (47\%) demonstrate RV hypertrophy confined to only the segments contiguous with the ventricular septum [37].

\section{Diastolic dysfunction}

In HCM, reduced ventricular volumes are frequent and the hyperkinetic appearance of systolic contraction translates into a normal or supernormal ejection fraction (EF) (> 65\%) until the end-stage of the disease [8]. EF is, therefore, considered inadequate to evaluate the indication for medical treatment and cardiac transplantation [2].

Diastolic dysfunction is one of the early hallmark features of HCM, and it is related to myocardial disarray and fibrosis, even in the absence of LV hypertrophy $[8,38]$.

Doppler parameters are accurate in evaluating diastolic function in HCM. Accurate measurements of transmitral and transpulmonary vein flow parameters using phase-contrast cardiac MRI are lower than those echocardiography, which is probably due to the lower temporal resolution of the breath-hold phase-contrast sequence. A new semi-automated, three-dimensional model-based analysis of the LV fillings curve showed a similar agreement with Doppler parameters [39].

In addition, myocardial tagging $[39,40]$ enables the quantification of cardiac strain evolution during late diastole, which can be used to assess diastolic myocardial dysfunction [40]. However, despite recent advances of myocardial tagging methods, the fading of taglines due to T1 relaxation is a common inherent limitation of all existing methods, which also requires prolonged imaging acquisition and post-processing times [39].

\section{Mitral valve apparatus}

Abnormalities in the morphology and function of the mitral valve apparatus are now considered a phenotypic expression of HCM that is independent of the hypertrophy distribution [11].

Mitral valve leaflets are increased in length in many HCM patients, and substantially elongated mitral valve leaflets are an important determinant of LVOT obstruction in some patients (Fig. 5) [8, 41].

Other abnormalities include hypertrophy of the papillary muscle heads, an increased number of papillary muscles, anterior and apical displacement of the papillary muscles, direct insertion of the anterolateral papillary muscle to the ventricular aspect of anterior mitral valve leaflet and LV accessory muscle bundles extending from the apex to the midventricular or basal levels of the LV anterior or septal walls (Fig. 6) [42].

\section{LV outflow tract obstruction}

LVOT obstruction is present in 70\% of classic HCM phenotype patients and is associated with an increased risk for heart failure-related death $[2,8]$. The mechanisms responsible for LVOT obstruction are related to complex anatomical relationships between the basal septum, LVOT, mitral valve, and papillary muscles $[8,41]$.

An elongated anterior mitral leaflet (Fig. 5a) is considered an important determination of LVOT obstruction, particularly in patients in whom the mitral leaflet length exceeds the transverse dimension of the outflow tract at end-systole 2-fold [3, $8,11]$. Systolic anterior motion (SAM) of the mitral valve refers to the paradoxical movement of the anterior leaflet and/or chordae toward the interventricular septum during systole. The precise mechanism of SAM in HCM remains unclear. Many investigators hypothesise that anterior mitral leaflet elongation and thickening and hypertrophy with anterior displacement of the papillary muscles contribute to the development of SAM. The resultant flow forces during systolic anterior motion pulling the leaflets into the LVOT in midand late-systole also result in reduced leaflet coaptation and a posteriorly directed mitral regurgitant jet (Fig. 5b) [25, 26].

Echocardiography remains the technique of choice to evaluate LVOT obstruction. However, cardiac MR-derived planimetry at the rest of the LVOT area during systole can identify obstructive HCM. A cutoff value of $2.7 \mathrm{~cm}^{2}$ has been reported as having an accuracy of $100 \%$ to differentiate obstructive from non-obstructive HCM [43]. Moreover, cine MRI in a long-axis cine view can provide a more accurate evaluation of the mechanism of outflow tract obstruction, demonstrating turbulent flow generated by systolic movement of the anterior mitral leaflet, chordae, and papillary muscle toward the interventricular septum [41] (Fig. 6).

\section{Left atrial size}

The left atrium (LA) is often enlarged in HCM patients, and its size provides important prognostic information. An enlarged LA is related to an increased morbidity and mortality in cardiovascular patients, and it is considered a marker for risk for SCD and potentially lethal arrhythmic events among HCM patients [44-46].

Volumetric MR quantification is the best approach to assess the LA size, but it may be too time-consuming. An area $\geqq$ $15 \mathrm{~cm}^{2} / \mathrm{m}^{2}$ and a transverse diameter $\geqq 2.8 \mathrm{~cm} / \mathrm{m}^{2}$ in the 


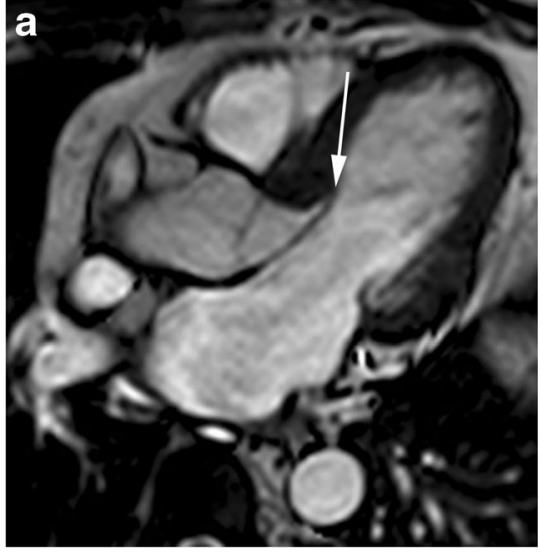

Fig. 5 Abnormalities of mitral apparatus in classic HCM phenotype. 44year-old woman with obstructive HCM, systolic anterior motion of the anterior leaflet of the mitral and mitral regurgitation. Horizontal long-axis SSFP cine MR images at (a) end-diastole and (b) mid-systole show an

four-chamber view are valuable alternatives to identify LA enlargement [47].

The cause of LA enlargement is multifactorial, but the most common mechanisms are SAM-related mitral regurgitation

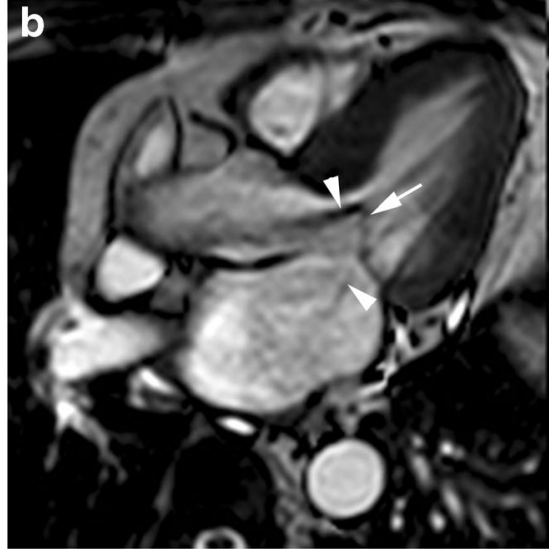

elongated anterior mitral leaflet (large arrow) with anterior displacement (small arrow) at mid-systole and turbulent-velocity jets (arrowheads) within the LV outflow tract and into the left atrium due to LV outflow tract obstruction and mitral regurgitation, respectively

and elevated LV filling pressures [48]. Mild or moderate LA dilatation is common and is related to LV diastolic dysfunction [44]. Severe LA dilattion is common when mitral regurgitation is associated with LVOT obstruction $[8,41]$.
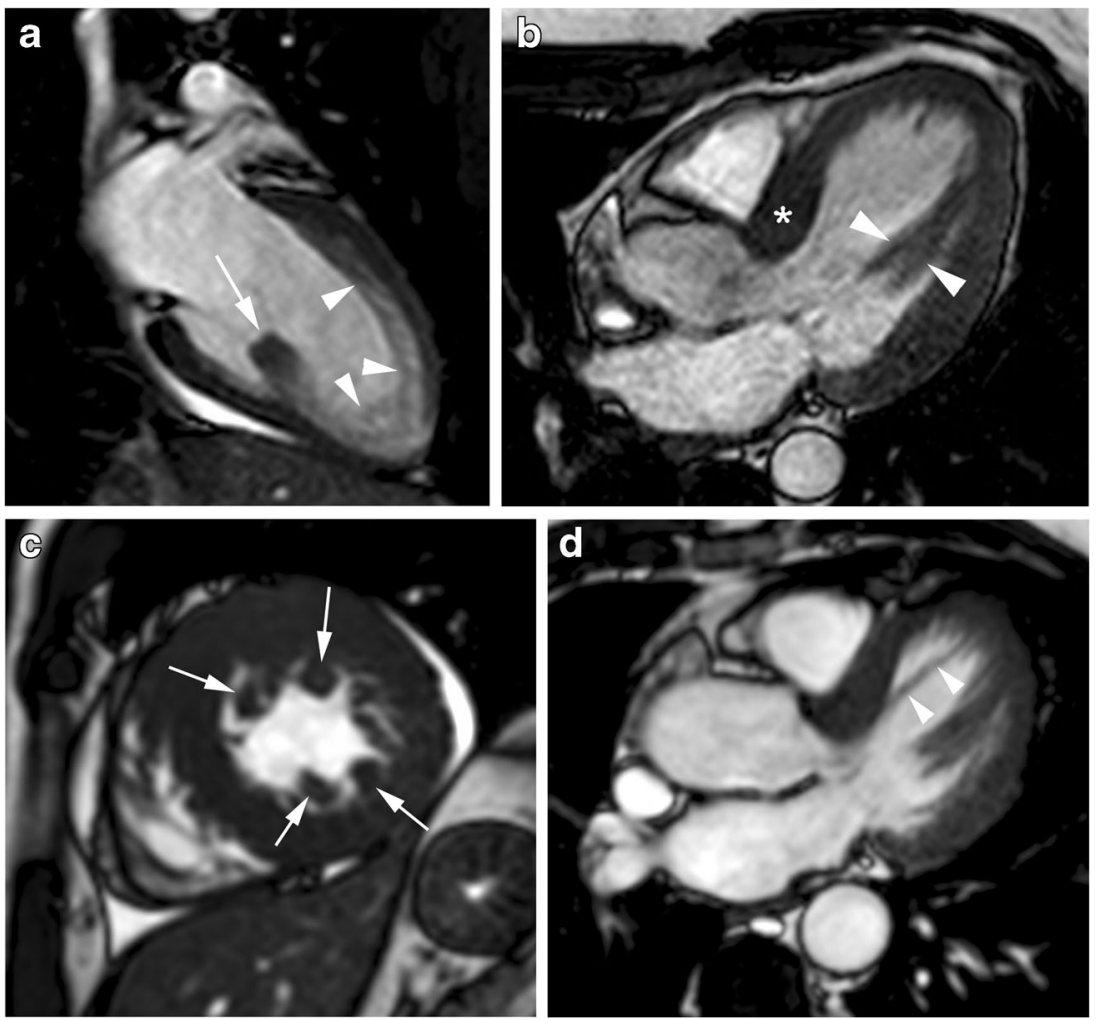

Fig. 6 Abnormalities of papillary muscles and mitral apparatus in classic HCM phenotype. a, 21-year-old woman with atypical chest and electrocardiographic abnormalities suggestive of HCM). End-diastolic twochamber SSFP cine MR image shows isolated posterior papillary muscle hypertrophy (arrow) and myocardial trabeculations along the anterior LV wall and apex (arrowheads); b, 45-year-old woman with asymmetric septal HCM and anomaly of papillary muscle. Horizontal long-axis SSFP cine MR image at end-diastole shows bifid anterolateral papillary

heads (arrowheads) and basal septal wall thickening (asterisk); c, 45-yearold man with chest pain. Short-axis SSFP cine MR image at end-diastole demonstrates multiple accessory papillary muscles, four in number (arrows), diffuse LV hypertrophy and pericardial effusion; d, 17-year-old man with myocardial hypertrophy limited to the basal ventricular septum. Horizontal long-axis SSFP cine MR image shows accessory left ventricular apical muscle bundle (arrowheads) traversing the LV cavity from the basal septal wall to the distal portion of the chamber 
Atrial fibrillation is the most common arrhythmia in HCM (20\%) and LA remodelling is the strongest predictor of atrial fibrillation independent of age and functional class [48].

\section{Microvascular ischaemia}

The hallmarks of HCM include LV hypertrophy, fibrosis and microvascular ischaemia, and whether there is a causative link between these features has not been determined to date [49].

Microvascular ischaemia in HCM, which is attributed to increased oxygen requirements due to hypertrophy, impaired ventricular relaxation, microvascular dysfunction of intramyocardial arterioles, and LVOT obstruction, is associated with chest pain, clinical deterioration, diastolic dysfunction, and an adverse prognosis [50,51]. It is most pronounced in the hypertrophied segments and in gene-positive HCM patients [52].

Although microvascular dysfunction may precede clinical deterioration for several years and is present in all stages of $\mathrm{HCM}$, it is not associated with evidence of permanent ischaemic damage and replacement fibrosis $[16,19]$.

The presence and severity of myocardial ischaemia and reduced myocardial blood flow can be diagnosed using noninvasive imaging modalities, including positron emission tomography (PET) [51], single photon emission computed tomography (SPECT) [53] and first-pass perfusion cardiac MR sequences $[8,54]$. Myocardial perfusion defects are usually found at subendocardium (most common) or at mid-wall, and it correlates with the areas of maximal wall thickness [54].

\section{Myocardial fibrosis}

Myocardial fibrosis in HCM is histopathologically diffuse in both the replacement and interstitial forms, and it is assumed to be a substrate for tachyarrhythmias and SCD. LGE essentially shows the focal distribution of fibrosis on cardiac MR, is present in less than half of those with the classic HCM phenotype and occupies a small percentage of the LV mass (median value: $2 \%$ ) [16, 55], suggesting that collagen deposition at this stage reflects an exaggerated activation of the matrix rather than a reparative process [56].

Myocardial LGE can present in a wide variety of patterns, although it never corresponds to a coronary vascular distribution (Figs. 4d and 7) $[15,57,58]$. It is usually localised in segments with the maximum LV wall thickness. Isolated or multiple patchy LGE at mid-wall (most common) (Fig. 7a, b) and along the RV insertion points on the septum (Fig. 7c) are the usual patterns of the classic HCM phenotype. LGE is unusual in non-hypertrophied segments $[8,59]$.

Histologically, fibrosis is often global, or diffuse, and often undetectable by standard LGE pulse sequences. T1 mapping is a novel and promising cardiac MR technique that provides an assessment of the total extent of the expanded extracellular space rather than the detection of regional areas of myocardial fibrosis identified by traditional LGE imaging. Patients with HCM have substantially higher-than-normal native T1 values and extracellular volume fractions, even in areas without LGE [60, 61] (Fig. 8), and reduced post-contrast myocardial T1 values consistent with the presence of diffuse interstitial fibrosis (Fig. 9). The imperfect concordance between native T1 mapping and LGE imaging for detecting focal replacement fibrosis may be explained by the distinct significance of both imaging methods because LGE (as well as postcontrast T1 mapping) reflects only the extracellular space, whereas native T1 mapping reflects a composite of both the intra- and extracellular compartments [62].

\section{Differential diagnosis}

Myocardial hypertrophy can occur as a result of a variety of cardiac and systemic diseases, such as hypertensive heart disease, amyloidosis and Anderson-Fabry disease [8, 63-65].
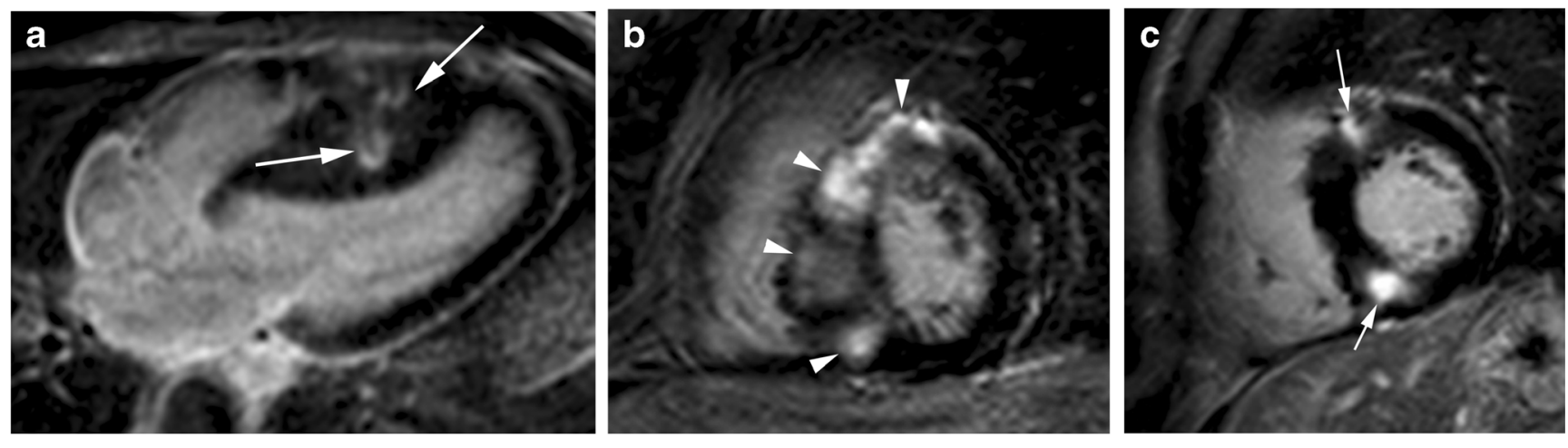

Fig. 7 Most common distribution of LGE in classic HCM phenotype. a, 16-year-old man with septal HCM and recurrent ventricular tachycardia. Four-chamber LGE image shows isolated patchy mid-wall septal enhancement (arrows); b, 65-year-old man with palpitations and syncope and diffuse HCM. Short-axis LGE image demonstrates diffuse patchy mid-wall septal enhancement in segments with maximum LV wall thickness (arrowheads); c, 48-year-old man with left bundle branch block at electrocardiography and asymmetrical septal. Short-axis LGE image shows patchy delayed enhancement at insertion points (arrows) of the $\mathrm{RV}$ wall into the anterior and posterior ventricular septum 


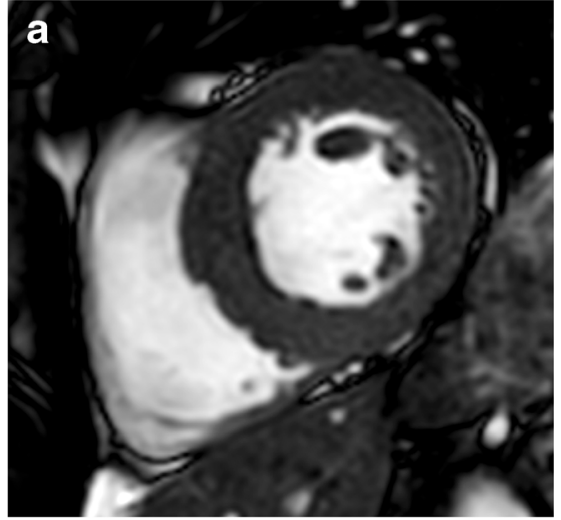

Fig. 8 T1 mapping in a 60-year-old man with septal HCM. a, Enddiastolic short-axis SSFP cine MR image showing septal myocardial hypertrophy $(20 \mathrm{~mm})$. b, Short-axis non-enhanced MOLLI (modified look locker inversion recovery) (1,5 T) T1 map demonstrates patchy areas of prolonged $\mathrm{T} 1$ values in the anteroseptal segment (arrowheads;

Systemic hypertension can lead to concentric LV hypertrophy, and discriminating between HCM and hypertensive heart disease is a frequent dilemma in clinical practice. Unlike $\mathrm{HCM}$, the LV wall thickness in hypertensive heart disease rarely exceeds $16 \mathrm{~mm}$, and usually, there is no LGE [63].

The differentiation of Anderson-Fabry disease from HCM remains a clinical challenge, given that there is overlap in their imaging findings. Recent studies have shown that native T1 values are significantly lower in patients with Anderson-Fabry disease compared with HCM patients (Fig. 10). This is probably the most sensitive and specific cardiac MR feature to differentiate between both diseases [64].

Hypertrophy in amyloidosis is usually moderate and relatively concentric, involving both ventricles, atria, inter-atrial septum and valves. Amyloidosis displays a specific pattern of global or segmental subendocardial LGE and frequently atrial enhancement as well [8]. In addition, the typical markedly elevated native $\mathrm{T} 1$ values contribute to differentiating amyloidosis from HCM [65].

\section{Risk stratification}

Although SCD is infrequent in classic HCM (0.5-1\%/year), the risk stratification for SCD should be performed in all patients with $\mathrm{HCM}$ due to the efficacy of the ICD in preventing SCD in this disease $[1,2,44]$.

The major risk factors for SCD in HCM include a family history of SCD, age (< 40 years), unexplained syncope, an abnormal blood pressure response to exercise, nonsustained ventricular tachycardia, a maximal LV wall thickness > $30 \mathrm{~mm}$, a LVOT gradient $\geq 30 \mathrm{mmHg}$ at rest and the LA diameter $[1,2,44]$. Although current risk factor models, such as the American algorithm [1] and European Society of Cardiology HCM Risk-SCD calculator [46] are highly
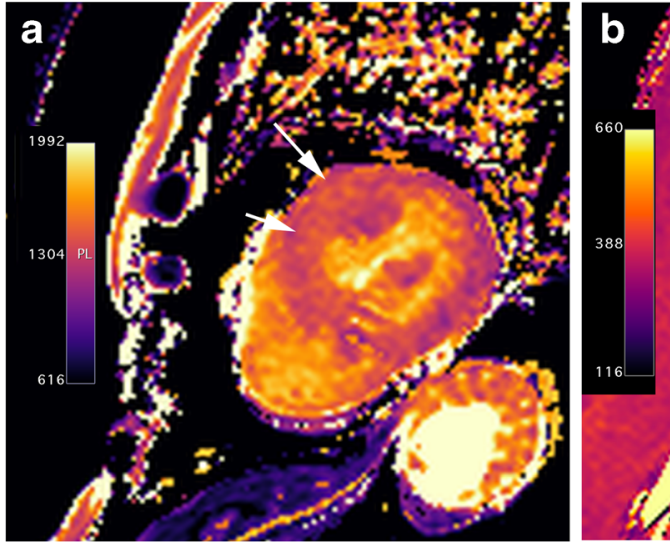

Fig. 9 T1 mapping in a 67-year-old woman with basal anterior and anteroseptal HCM. a, Short-axis nonenhanced MOLLI (1.5 T) T1 map shows prolonged $\mathrm{T} 1$ values in the area of myocardial hypertrophy (arrows). b, T1 map obtained at LGE MR imaging shows abnormal shortening of the T1 values in the hypertrophied segments (arrowheads). The

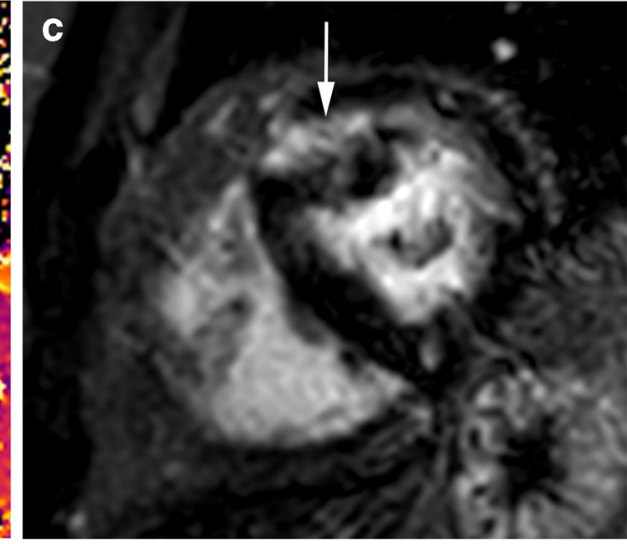

extracellular volume (ECV) for the hypertrophied segments was abnormally elevated (44,6 vs $29 \%$ for the lateral wall) (haematocrit, 0.42). Normal ECV values of $25.3 \pm 3.5 \%$ (1.5 T). c, LGE MR image through the same plane shows midwall enhancement in the hypertrophied segments (arrow) 
Fig. 10 45-year-old man with gene-positive Anderson-Fabry disease. a, End-diastolic fouchamber SSFP cine MR image shows diffuse and symmetric LV wall hypertrophy (asterisks). b, Non-enhanced short-axis MOLLI native $\mathrm{T} 1 \mathrm{map}$ at $1.5 \mathrm{~T}$ shows significant shortening of $\mathrm{T} 1$ values ( $840 \mathrm{~ms})$ in the hypertrophy segments. Post-gadolinium images were not obtained by renal impairment
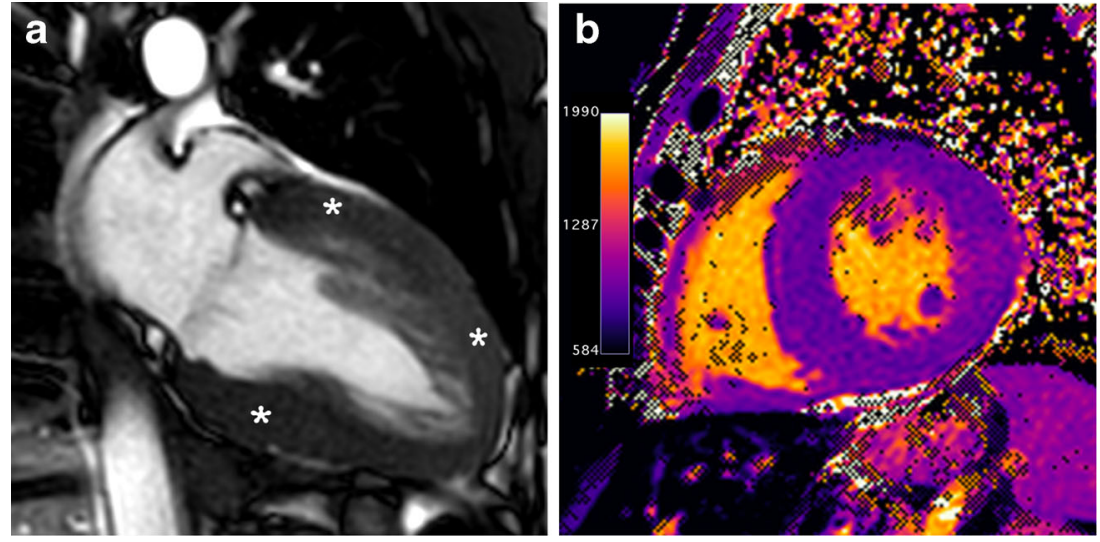

effective in identifying many patients with HCM who will benefit from ICD, some patients without conventional risk markers nevertheless remain at risk for SCD [66].

Currently, neither American guidelines nor the HCM RiskSCD calculator incorporate LGE $[1,44]$. However, multiple studies have demonstrated that LGE is correlated with areas of myocardial fibrosis as the arrhythmogenic substrate for ventricular tachyarrhythmias $[27,46,67]$ and might be considered a potential risk modifier, particularly when it is seen in extensive areas of the myocardium (LGE $\geq 15 \%$ of the LV myocardium) $[13,14,66,68]$. It has been postulated that $\mathrm{T} 1$ mapping may prove to be superior to LGE for risk stratification in HCM. However, to date, there has been no link between T1 mapping and cardiovascular outcomes within HCM [69].

Another imaging feature with a potential impact on the current risk stratification models could be the myocardial high T2 signal intensity areas. These areas have been related to markers of adverse disease progression, such as LGE, elevated troponin and non-sustained ventricular tachycardia [70] and they have been recently implicated as a potential tool for the prediction of SCD [71].

\section{Adverse remodelling}

Adverse remodelling occurs in 15-20\% of HCM patients and is characterised by progressive decreases in systolic (EF: 50$65 \%$ ) and diastolic LV function [16, 55], moderate/severe LA and LV dilatation, an increase in symptoms and functional limitations [72], atrial fibrillation [38], reduction or loss of LVOT, and progressive LV wall thinning, which are thought to be due to small-vessel ischaemia (Fig. 11) [73].

Myocardial LGE is common (> 50\%) [8] and is usually multiple and confluent at the mid-wall or transmural $[8,16]$, and the extent of LGE accounts for $10-15 \%$ or more of the LV mass $[8,14]$.

The risk for $\mathrm{SCD}$ is intermediate (3-5\%/year) ranging from a low risk for the classic HCM and a high risk for the endstage $\operatorname{HCM}[8,15,16]$.

The diagnosis of adverse remodelling is important because it can alter management strategies including the consideration of prophylactic ICD, and timely evaluation for heart transplantation, before symptoms develop [72].

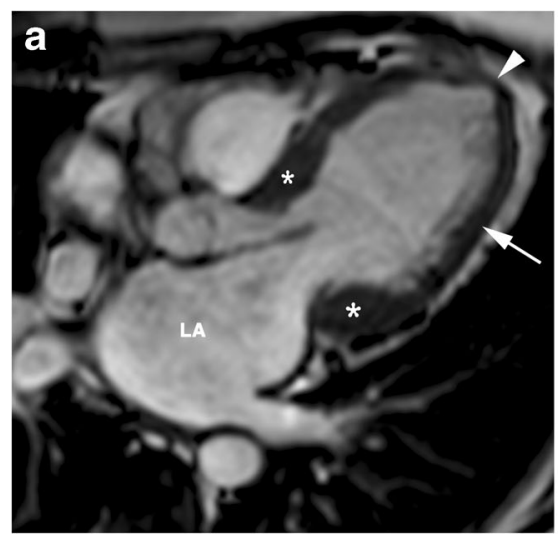

Fig. 11 Adverse remodelling. 77-year-old man with long-standing asymmetric septal and lateral HCM who presented with increasing dyspnea on exertion and palpitations. a, End-diastolic horizontal long-axis SSFP cine MR image shows dilated and dysfunctional LV (ejection fraction, 45\%), moderately thickened basal septum and lateral wall (15 mm) (asterisks),

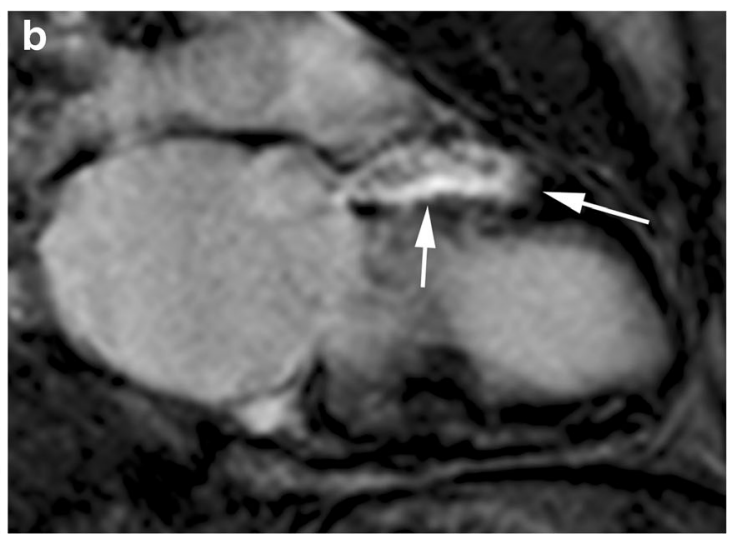

substantial thinning of the lateral LV wall (arrow), apical aneurysm (arrowhead) and dilated left atrium (LA). b, Short-axis inversion-recovery image shows transmural delayed enhancement in the anterior wall (arrows) 


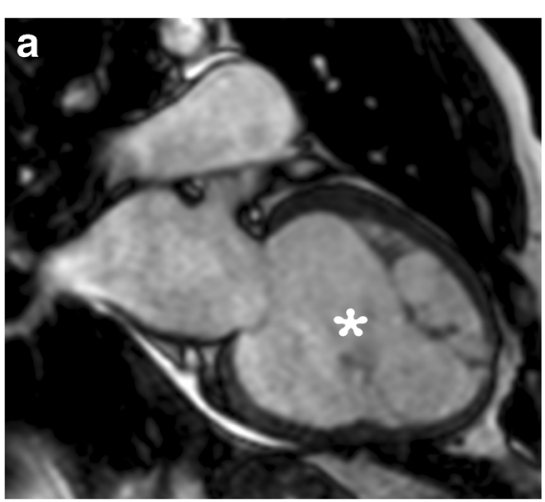

Fig. 12 End-stage HCM with dilated form. 69-year-old man with longstanding asymmetric septal HCM who presented with dyspnea at rest and congestive heart failure. a, Two-chamber SSFP cine MR image shows regression of wall thickness, increase in left ventricular cavity dimensions

\section{End-stage HCM}

A small distinctive group of HCM patients (5-10\%) will develop the high-risk phenotype of end-stage disease, which is characterised by progressive LV wall thinning, LV dilatation, a decrease or disappearance of LVOT gradients and LV systolic $(\mathrm{EF}<50 \%)$ and diastolic dysfunction $[8,16,62,73]$.

In certain cases, there is relative LV dilation and wall thinnings, with greater resemblance to the morphological and functional features of dilated cardiomyopathy (Fig. 12). In other cases, a restrictive pattern with a small LV and severe dilatation of both atria occurs (Fig. 13) $[2,8]$.

Most end-stage HCM patients show mid-wall or transmural LGE $(75-100 \%)[8,14]$. The extent of LGE often exceeds that observed in any other cardiovascular disease ( $>25 \%$ of the LV mass) [27], and it is more extensive in those

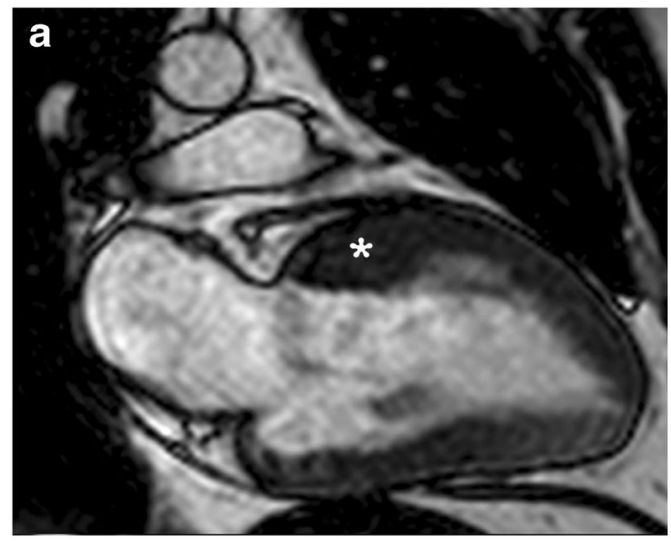

Fig. 13 End stage HCM with restrictive pattern. 77-year-old man who presented with several months of intermittent exertional dyspnea and asymmetric HCM. He presented to the emergency room 7 years later with markedly worsening dyspnea and decompensated heart failure. a Twochamber SSFP cine MR image performed at initial diagnosis shows

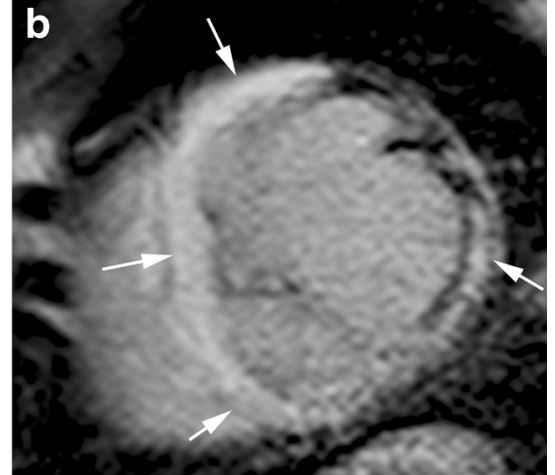

(asterisk) and a reduced ejection-fraction of $33 \%$. b. Short-axis inversionrecovery image showing transmural (arrows) delayed enhancement involving more than $25 \%$ of myocardial mass

with ventricular remodelling and the LA is larger in those with a normal ventricular size $[8,27,72]$.

This end-stage form is associated with an increased risk for SCD (by $10 \% /$ year) and heart failure-related complications $[8$, $16]$.

\section{Conclusions}

The phenotypic expression of HCM includes a wide variety of morphological and functional manifestations that may lead to severe left ventricular wall remodelling.

Cardiac MRI provides the clinician with detailed information regarding the HCM phenotypes and enables the assessment of its functional consequences elucidating the causes and site of hypothetical dynamic obstruction, the presence and extent of myocardial perfusion abnormalities and the existence of fibrosis.

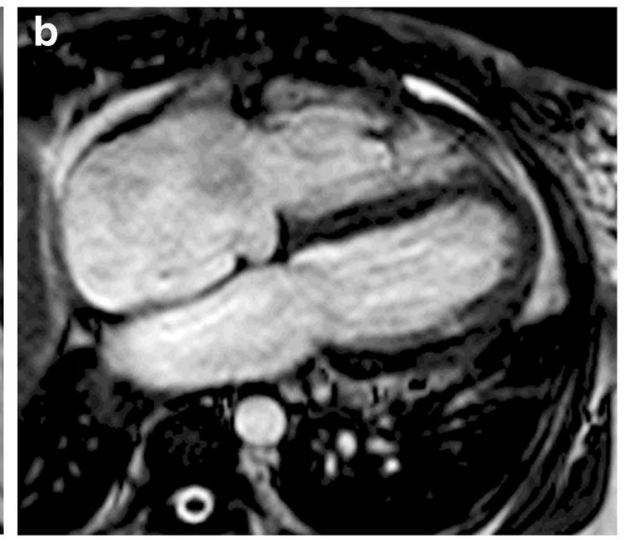

asymmetric anterior hypertrophy (asterisk) with an ejection-fraction of $60 \%$. b, Four-chamber SSFP cine MR image performed 7 years later demonstrates a small LV cavity, atrial cavities enlargement, regression of wall thickness and a reduced ejection-fraction of $37 \%$ 
Acknowledgements This review was presented in part as an educational exhibit at the 2016 RSNA Scientific Assembly, Chicago IL as: Cristina Méndez, Susana A Otero Muinelo, Tania Pérez Ramos, Rafaela Soler, Esther Rodríguez, Roberto Barriales Villa, Juan Pablo Ochoa, Lorenzo Montserrat Iglesias. MRI of Hypertrophic Cardiomyopathy: Phenotypes and Phenocopies. Abstract available at: archive.rsna.org/2016/16013821.html

Funding R. Barriales-Villa and Lorenzo Monserrat are part of a cardiovascular research network CIBER in Cardiovascular Diseases (CB16/11/ 00425).

Open Access This article is distributed under the terms of the Creative Commons Attribution 4.0 International License (http:// creativecommons.org/licenses/by/4.0/), which permits unrestricted use, distribution, and reproduction in any medium, provided you give appropriate credit to the original author(s) and the source, provide a link to the Creative Commons license, and indicate if changes were made.

\section{References}

1. American College of Cardiology Foundation/American Heart Association Task Force on Practice, American Association for Thoracic Surgery, American Society of Echocardiography et al (2011) ACCF/AHA guideline for the diagnosis and treatment of hypertrophic cardiomyopathy: a report of the american college of cardiology foundation/american heart association task force on practice guidelines. J Thorac Cardiovasc Surg 142:e153-e203

2. Authors/Task Force members, Elliott PM, Anastasakis A et al (2014) ESC guidelines on diagnosis and management of hypertrophic cardiomyopathy: the task force for the diagnosis and management of hypertrophic cardiomyopathy of the european society of cardiology. Eur Heart J 35:2733-2779

3. Baxi AJ, Restrepo CS, Vargas D, Marmol-Velez A, Ocazionez D, Murillo H (2016) Hypertrophic cardiomyopathy from a to Z: genetics, pathophysiology, imaging, and management. Radiographics 36:335-354

4. Lopes LR, Zekavati A, Syrris P et al (2013) Genetic complexity in hypertrophic cardiomyopathy revealed by high-throughput sequencing. J Med Genet 50:228-239

5. Maron BJ, Maron MS, Semsarian C (2012) Genetics of hypertrophic cardiomyopathy after 20 years: clinical perspectives. J Am Coll Cardiol 60:705-715

6. Hughes SE (2004) The pathology of hypertrophic cardiomyopathy. Histopathology 44:412-427

7. Maron BJ, Gardin JM, Flack JM, Gidding SS, Kurosaki TT, Bild DE (1995) Prevalence of hypertrophic cardiomyopathy in a general population of young adults. Echocardiographic analysis of 4111 subjects in the CARDIA study. Coronary artery risk development in (young) adults. Circulation 92:785-789

8. Bogaert J, Olivotto I (2014) MR imaging in hypertrophic cardiomyopathy: from magnet to bedside. Radiology 273:329-348

9. Efthimiadis GK, Pagourelias ED, Hadjimiltiades S, Meditskou S, Karvounis H, McKenna WJ (2015) Feasibility and significance of preclinical diagnosis in hypertrophic cardiomyopathy. Cardiol Rev 23:297-302

10. Moon JC, McKenna WJ (2012) Myocardial crypts: a prephenotypic marker of hypertrophic cardiomyopathy? Circ Cardiovasc Imaging 5:431-432

11. Maron MS, Olivotto I, Harrigan C et al (2011) Mitral valve abnormalities identified by cardiovascular magnetic resonance represent a primary phenotypic expression of hypertrophic cardiomyopathy. Circulation 124:40-47
12. Captur G, Lopes LR, Patel V et al (2014) Abnormal cardiac formation in hypertrophic cardiomyopathy: fractal analysis of trabeculae and preclinical gene expression. Circ Cardiovasc Genet 7:241-248

13. Choudhury L, Rigolin VH, Bonow RO (2017) Integrated imaging in hypertrophic cardiomyopathy. Am J Cardiol 119:328-339

14. Chan RH, Maron BJ, Olivotto I et al (2014) Prognostic value of quantitative contrast-enhanced cardiovascular magnetic resonance for the evaluation of sudden death risk in patients with hypertrophic cardiomyopathy. Circulation 130:484-495

15. O'Hanlon R, Grasso A, Roughton M et al (2010) Prognostic significance of myocardial fibrosis in hypertrophic cardiomyopathy. $\mathrm{J}$ Am Coll Cardiol 56:867-874

16. Olivotto I, Cecchi F, Poggesi C, Yacoub MH (2012) Patterns of disease progression in hypertrophic cardiomyopathy: an individualized approach to clinical staging. Circ Heart Fail 5(4):535-546

17. Ho CY, Abbasi SA, Neilan TG et al (2013) T1 measurements identify extracellular volume expansion in hypertrophic cardiomyopathy sarcomere mutation carriers with and without left ventricular hypertrophy. Circ Cardiovasc Imaging 6:415-422

18. Rowin EJ, Maron MS, Lesser JR, Maron BJ (2012) CMR with late gadolinium enhancement in genotype positive-phenotype negative hypertrophic cardiomyopathy. JACC Cardiovasc Imaging 5:119122

19. Maron MS, Maron BJ (2015) Clinical impact of contemporary cardiovascular magnetic resonance imaging in hypertrophic cardiomyopathy. Circulation 132:292-298

20. Germans T, Wilde AA, Dijkmans PA et al (2006) Structural abnormalities of the inferoseptal left ventricular wall detected by cardiac magnetic resonance imaging in carriers of hypertrophic cardiomyopathy mutations. J Am Coll Cardiol 48:2518-2513

21. Deva DP, Williams LK, Care M et al (2013) Deep basal inferoseptal crypts occur more commonly in patients with hypertrophic cardiomyopathy due to disease causing myofilament mutations. Radiology 269:68-76

22. Maron MS, Rowin EJ, Lin D et al (2012) Prevalence and clinical profile of myocardial crypts in hypertrophic cardiomyopathy. Circ Cardiovasc Imaging 5:441-447

23. Cresti A, Cannarile P, Aldi E et al (2018) Multimodality imaging and clinical significance of congenital ventricular outpouchings: recesses, diverticula, aneurysms, clefts, and crypts. J Cardiovasc Echogr 28:9-17

24. Basso C, Marra P, Thiene G (2014) Myocardial clefts, crypts, or crevices once again, you see only what you look for. Circ Cardiovasc Imaging 17:217-219

25. Jiang L, Levine RA, King ME, Weyman AE (1987) An integrated mechanism for systolic anterior motion of the mitral valve in hypertrophic cardiomyopathy based on echocardiographic observations. Am Heart J 113:633-644

26. Klues HG, Proschan MA, Dollar AL, Spirito P, Roberts WC, Maron BJ (1993) Echocardiographic assessment of mitral valve size in obstructive hypertrophic cardiomyopathy. Anatomic validation from mitral valve specimen. Circulation 88:548-555

27. Maron MS (2012) Clinical utility of cardiovascular magnetic resonance in hypertrophic cardiomyopathy. J Cardiovasc Magn Reson $14: 13$

28. Tarkiainen M, Sipola P, Jalanko M et al (2016) Cardiovascular magnetic resonance of mitral valve length in hypertrophic cardiomyopathy. J Cardiovasc Magn Reson 18:33

29. Oechslin E, Jenni R (2011) Left ventricular non-compaction revisited: a distinct phenotype with genetic heterogeneity? Eur Heart J 32:1446-1456

30. Petersen SE, Selvanayagam JB, Francis JM et al (2005) Differentiation of athlete's heart from pathological forms of cardiac hypertrophy by means of geometric indices derived from cardiovascular magnetic resonance. J Cardiovasc Magn Reson 7:551558 
31. Maron BJ, Pelliccia A, Spirito P (1995) Cardiac disease in young trained athletes. Insights into methods for distinguishing athlete's heart from structural heart disease, with particular emphasis on hypertrophic cardiomyopathy. Circulation 91:1596-1601

32. Swoboda PP, McDiarmid AK, Erhayiem B et al (2016) Assessing myocardial extracellular volume by T1 mapping to distinguish hypertrophic cardiomyopathy from athlete's heart. J Am Coll Cardiol 67:2189-2190

33. Maron BJ, Spirito P, Wesley Y, Arce J (1986) Development and progression of left ventricular hypertrophy in children with hypertrophic cardiomyopathy. N Engl J Med 315:610-614

34. Moon JC, Fisher NG, McKenna WJ, Pennell DJ (2004) Detection of apical hypertrophic cardiomyopathy by cardiovascular magnetic resonance in patients with non-diagnostic echocardiography. Heart 90:645-649

35. Maron MS, Maron BJ, Harrigan C et al (2009) Hypertrophic cardiomyopathy phenotype revisited after 50 years with cardiovascular magnetic resonance. J Am Coll Cardiol 54:220-228

36. Maron MS, Finley JJ, Bos JM et al (2008) Prevalence, clinical significance, and natural history of left ventricular apical aneurysms in hypertrophic cardiomyopathy. Circulation 118:1541-1549

37. Maron MS, Hauser TH, Dubrow E et al (2007) Right ventricular involvement in hypertrophic cardiomyopathy. Am J Cardiol 100: 1293-1298

38. Tsang TS, Barnes ME, Gersh BJ, Bailey KR, Seward JB (2002) Left atrial volume as a morphophysiologic expression of left ventricular diastolic dysfunction and relation to cardiovascular risk burden. Am J Cardiol 90:1284-1289

39. Wu V, Chyou JY, Chung S, Bhagavatula S, Axel L (2014) Evaluation of diastolic function by three-dimensional volume tracking of the mitral annulus with cardiovascular magnetic resonance: comparison with tissue Doppler imaging. J Cardiovasc Magn Reson 16:71

40. Shehata ML, Cheng S, Osman NF, Bluemke DA, Lima JA (2009) Myocardial tissue tagging with cardiovascular magnetic resonance. J Cardiovasc Magn Reson 11:55

41. Patel P, Dhillon A, Popovic ZB et al (2015) Left ventricular outflow tract obstruction in hypertrophic cardiomyopathy patients without severe septal hypertrophy: implications of mitral valve and papillary muscle abnormalities assessed using cardiac magnetic resonance and echocardiography. Circ Cardiovasc Imaging 8:e003132

42. Gruner C, Chan RH, Crean A et al (2014) Significance of left ventricular apical-basal muscle bundle identified by cardiovascular magnetic resonance imaging in patients with hypertrophic cardiomyopathy. Eur Heart J 35:2706-2713

43. Schulz-Menger J, Abdel-Aty H, Busjahn A et al (2006) Left ventricular outflow tract planimetry by cardiovascular magnetic resonance differentiates obstructive from non-obstructive hypertrophic cardiomyopathy. J Cardiovasc Magn Reson 8:741-746

44. O'Mahony C, Jichi F, Pavlou M et al (2014) A novel clinical risk prediction model for sudden cardiac death in hypertrophic cardiomyopathy (HCM risk-SCD). Eur Heart J 35:2010-2020

45. Nistri S, Olivotto I, Betocchi S et al (2006) Prognostic significance of left atrial size in patients with hypertrophic cardiomyopathy (from the Italian registry for hypertrophic cardiomyopathy). Am J Cardiol 98:960-965

46. Spirito P, Autore C, Formisano F et al (2014) Risk of sudden death and outcome in patients with hypertrophic cardiomyopathy with benign presentation and without risk factors. Am J Cardiol 113: $1550-1555$

47. Maceira AM, Cosín-Sales J, Roughton M, Prasad SK, Pennell DJ (2010) Reference left atrial dimensions and volumes by steady state free precession cardiovascular magnetic resonance. J Cardiovasc Magn Reson 12:65
48. Olivotto I, Cecchi F, Casey SA, Dolara A, Traverse JH, Maron BJ (2001) Impact of atrial fibrillation on the clinical course of hypertrophic cardiomyopathy. Circulation 104:2517-2524

49. Maron MS, Olivotto I, Maron BJ et al (2009) The case for myocardial ischemia in hypertrophic cardiomyopathy. J Am Coll Cardiol 54:866-875

50. Knaapen P, Germans T, Camici PG et al (2008) Determinants of coronary microvascular dysfunction in symptomatic hypertrophic cardiomyopathy. Am J Physiol Heart Circ Physiol 294:H986-H993

51. Cecchi F, Olivotto I, Gistri R, Lorenzoni R, Chiriatti G, Camici PG (2003) Coronary microvascular dysfunction and prognosis in hypertrophic cardiomyopathy. N Engl J Med 349:1027-3105

52. Olivotto I, Girolami F, Sciagrà R et al (2011) Microvascular function is selectively impaired in patients with hypertrophic cardiomyopathy and sarcomere myofilament gene mutations. J Am Coll Cardiol 58:839-848

53. Romero-Farina G, Candell-Riera J, Galve E et al (2004) Do myocardial perfusion SPECT and radionuclide angiography studies in adult patients with hypertrophic cardiomyopathy have prognostic implications? J Nucl Cardiol 11:578-586

54. Petersen SE, Jerosch-Herold M, Hudsmith LE et al (2007) Evidence for microvascular dysfunction in hypertrophic cardiomyopathy: new insights from multiparametric magnetic resonance imaging. Circulation 115:2418-2425

55. Olivotto I, Maron BJ, Appelbaum E et al (2010) Spectrum and clinical significance of systolic function and myocardial fibrosis assessed by cardiovascular magnetic resonance in hypertrophic cardiomyopathy. Am J Cardiol 106:261-267

56. Ho CY, López B, Coelho-Filho OR et al (2010) Myocardial fibrosis as an early manifestation of hypertrophic cardiomyopathy. N Engl J Med 363:552-563

57. Bruder O, Wagner A, Jensen CJ et al (2010) Myocardial scar visualized by cardiovascular magnetic resonance imaging predicts major adverse events in patients with hypertrophic cardiomyopathy. J Am Coll Cardiol 56:875-887

58. Rubinshtein R, Glockner JF, Ommen SR et al (2010) Characteristics and clinical significance of late gadolinium enhancement by contrast-enhanced magnetic resonance imaging in patients with hypertrophic cardiomyopathy. Circ Heart Fail 3:5158

59. Tyan CC, Armstrong S, Scholl D et al (2013) Stress hypoperfusion and tissue injury in hypertrophic cardiomyopathy: spatial characterization using high-resolution 3-tesla magnetic resonance imaging. Circ Cardiovasc Imaging 6:229-238

60. Puntmann VO, Voigt T, Chen $\mathrm{Z}$ et al (2013) Native T1 mapping in differentiation of normal myocardium from diffuse disease in hypertrophic and dilated cardiomyopathy. JACC Cardiovasc Imaging 6:475-484

61. Dass S, Suttie JJ, Piechnik SK et al (2012) Myocardial tissue characterization using magnetic resonance noncontrast $\mathrm{t} 1$ mapping in hypertrophic and dilated cardiomyopathy. Circ Cardiovasc Imaging 5:726-733

62. Sado DM, Flett AS, Banypersad SM et al (2012) Cardiovascular magnetic resonance measurement of myocardial extracellular volume in health and disease. Heart 98:1436-1441

63. Rodrigues JC, Rohan S, Ghosh Dastidar A et al (2017) Hypertensive heart disease versus hypertrophic cardiomyopathy: multi-parametric cardiovascular magnetic resonance discriminators when end-diastolic wall thickness $\geq 15 \mathrm{~mm}$. Eur Radiol 27:11251135

64. Sado DM, White SK, Piechnik SK et al (2013) Identification and assessment of Anderson-Fabry disease by cardiovascular magnetic resonance noncontrast myocardial T1 mapping. Circ Cardiovasc Imaging 6:392-398 
65. Fontana M, Banypersad SM, Treibel TA et al (2014) Native T1 mapping in transthyretin amyloidosis. JACC Cardiovasc Imaging 7:157-165

66. Maron BJ, Maron MS (2016) LGE means better selection of HCM patients for primary prevention implantable defibrillators. JACC Cardiovasc Imaging 9:1403-1406

67. Christiaans I, van Engelen K, van Langen IM et al (2010) Risk stratification for sudden cardiac death in hypertrophic cardiomyopathy: systematic review of clinical risk markers. Europace 12:313321

68. He D, Ye M, Zhang L, Jiang B (2018) Prognostic significance of late gadolinium enhancement on cardiac magnetic resonance in patients with hypertrophic cardiomyopathy. Heart Lung 47:122126

69. Rowin EJ, Maron MS (2016) The role of cardiac MRI in the diagnosis and risk stratification of hypertrophic cardiomyopathy. Arrhythm Electrophysiol Rev 5:197-202

70. Todiere G, Pisciella L, Barison A et al (2014) Abnormal T2-STIR magnetic resonance in hypertrophic cardiomyopathy: a marker of advanced disease and electrical myocardial instability. PLoS One 9: e111366
71. Gommans DHF, Cramer GE, Bakker J et al (2018) High T2weighted signal intensity for risk prediction of sudden cardiac death in hypertrophic cardiomyopathy. Int J Cardiovasc Imaging 34:113120

72. Harris KM, Spirito P, Maron MS et al (2006) Prevalence, clinical profile, and significance of left ventricular remodeling in the endstage phase of hypertrophic cardiomyopathy. Circulation 114:216 225

73. Maron BJ, Spirito P (1998) Implications of left ventricular remodeling in hypertrophic cardiomyopathy. Am J Cardiol 81:1339-1344

\section{Publisher's Note}

Springer Nature remains neutral with regard to jurisdictional claims in published maps and institutional affiliations. 